\section{FREE CPD ON GUM DISEASE}

GSK, manufacturers of Corsodyl, have launched a new distance learner for dental professionals on the topic of gum disease.

According to the Delivering better oral health toolkit, maintaining periodontal health and preventing periodontitis should be based on detecting periodontitis early using the Basic Periodontal Examination (BPE) and managing the factors that expose patients to a greater risk of the disease, eg smoking, diabetes and medications.

The 2009 Adult Dental Health Survey found that only $17 \%$ of dentate adults in England, Wales and Northern Ireland had very healthy periodontal tissue and no periodontal disease. This confirms a need for continued patient education regarding gum health.

The Corsodyl distance learner module provides training on periodontal disease, the BPE, and patient management to treat and prevent the condition. It is suitable for the whole dental team to use and is available 24 hours a day. On top of this, there is no time limit to complete this module and completion of the module can contribute up to 1.5 hours towards your verifiable CPD. www.gsk-dentalprofessionals.co.uk

\section{IS THE NORTH-SOUTH DIVIDE FACT OR FICTION?}

During the last few years it has been factually proven that London and the South East has achieved significantly higher values and multipliers of profit than any other part of the country. However, when these areas are excluded from Christie \& Co's analysis, the north-south divide is less evident with values across the country seeming relatively similar save for marginal wage variations and specialisms.

In the last six months Christie \& Co has looked at over 45 practices (for banking purposes) with an approximate value in excess of $£ 25$ million within the northern region of the country where values achieved have been extremely strong for all types of practices.

Christie \& Co often see achieved values being within the market guidelines/average, both as a multiplier of $\mathrm{Net}$ Annual Adjusted Profit (EBITDA) and as a percentage in the pound, although within certain circumstances multipliers and percentages are at significantly higher levels depending on the demand of the practice in question.

The market within the northern portion of the country is considered to be extremely strong for all practices with a particular emphasis on NHS-driven income although private practices are seeing more demand and as a result higher values are beginning to be achieved on a more regular basis.

Again it is common where a private practice has high insurance backed income (which creates less risk for a new purchaser) for values to exceed the industry norm.

To discuss any matters relating to your dental practice, visit www.christie.com.

\title{
A PIVOTAL MOMENT IN PATIENT MARKETING
}

Software of Excellence's newly launched EXACT V12 signals a pivotal moment in dentistry, as for the first time it presents the means by which to seamlessly connect marketing and business functions. Using software enhancements dentists will be able to truly understand the return on investment achieved from their marketing activity and take full advantage of new digital communication platforms, helping to attract more new patients and increase revenue.

EXACT V12 includes brand new modules that enable practices to optimise online search engines and to widely distribute positive patient recommendations which will benefit their website ranking. The new Marketing Manager module enables practices to send targeted, automated communications to specific patient lists and instantly track results; including number of phone enquiries, appointments booked and treatment uptake. Practices can then delve even deeper by allocating unique telephone numbers to campaigns, which via the Channel Track module, records calls and monitors which communications are most effective. This ability to accurately monitor results and calculate return on investment (ROI) is a major step forward for practices.

EXACT V12's focus on patient marketing now enables dental practices to take control of an automated patient communication plan, optimising their online presence and using digital platforms to maximum effect as part of a fully co-ordinated marketing strategy.

Visit www.softwareofexcellence. com or telephone 08453455767.

\section{EFFECTIVE TISSUE REGENERATION AND IMPLANT SUCCESS}

Nobel Biocare's latest solution for use in guided bone and tissue regeneration procedures is the creos xenoprotect, composed of a network of highly purified porcine collagen and elastin fibres, interwoven to form a dense mesh.

This biodegradable noncrosslinked collagen membrane has excellent handling properties. Compared to competitor products, creos xenoprotect can be cut or pre-shaped when dry according to the size of the individual defect. Its hydrophilic nature ensures that the hydration process takes only seconds, and with no functionally preferred side, the membrane can be placed on either side to facilitate ease of use.

Due to a minimal increase in size when moist, you do not have to make allowances for expansion - what you cut off is what you use, making it very cost-effective. The membrane is also easy to unfold and reposition even when hydrated, enabling you to consistently achieve excellent results while saving you

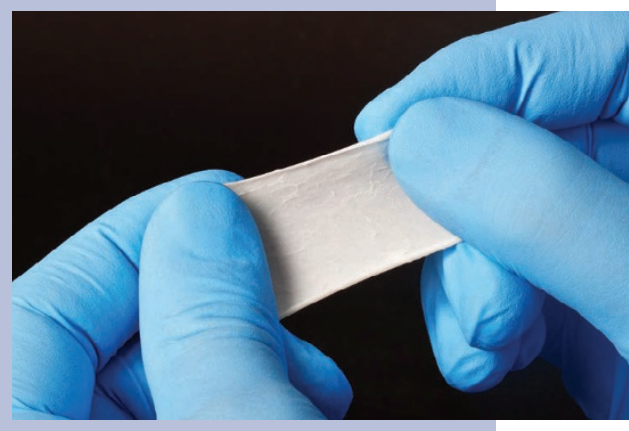

time and money. With three different size membranes - $15 \times 20 \mathrm{~mm}$ (small), $25 \times 30 \mathrm{~mm}$ (medium) and $30 \times 40 \mathrm{~mm}$ (large) - available, wastage is reduced.

Enabling you to make better use of your time and enhancing the patient experience, the membrane is resorbable requiring no further surgery for its removal. Designed to resorb safely over a prolonged degradation time, the aesthetic outcome is improved, providing convenience for you and your patient.

To arrange for a FREE demo or for more information on creos xenoprotect from Nobel Biocare, call 0208 7563300 or visit www.nobelbiocare. com. 